Euskal ikerketen aldizkaria | Revue d'études basques |

Revista de estudios vascos | Basque studies review

$6 \mid 2001$

Numéro VI

\title{
Catholicisme et construction identitaire basque : retour sur le postulat d'une sécularisation achevée
}

Xabier Itçaina

\section{OpenEdition}

\section{Journals}

Édition électronique

URL : http://journals.openedition.org/lapurdum/1239

DOI : 10.4000/lapurdum.1239

ISSN : 1965-0655

Éditeur

IKER

Édition imprimée

Date de publication : 1 octobre 2001

Pagination : 353-366

ISBN : 2-84127-156-0

ISSN : $1273-3830$

Référence électronique

Xabier Itçaina, "Catholicisme et construction identitaire basque : retour sur le postulat d'une sécularisation achevée », Lapurdum [En ligne], 6 | 2001, mis en ligne le 01 juin 2009, consulté le 31 janvier 2020. URL : http://journals.openedition.org/lapurdum/1239; DOI : 10.4000/lapurdum.1239 


\section{Xabier Itçaina}

CNRS-CERVL, Pouvoir, Action publique, Territoire IEP Bordeaux

x.itcaina@iep.u-bordeaux.fr

\section{Catholicisme et construction identitaire basque : retour sur le postulat d'une sécularisation achevée}

La recherche présentée ici I entend « détourner » quatre approches du lien entre religion et identité collective : Ie récit essentialiste (il y a une fusion entre appartenances religieuse et ethnique), le récit exégétique ou théologique (il y a une spiritualité iclentitaire spécifíque, qui requiert un ajustement normatif du religieux), le récit instrumentaliste (la religion, forme déguisée de l'idéologie, est une structure de domination parmi d'autres) et le récit annexionniste (tout est religicux : les mouvements identitaires ne sont que des religions séculières, qui se sont substituéci à un système de pratiques et de croyances en perte de vitesse). A partir d'une vision à la fois plus constructiviste et plus compréhensive des identités catholique et basque, la thèse propose un cinquième récit.

La recherche a été guidée par la question suivante : dans quelle mesure l'interaction entre les deux activités identitaires catholique et basque a-t-elle effectivement génếré des objectivations et des subjectivations spécifiquess ayant en retour des effets structurants sur ces deux types de mobilisations'? En guise d'hypothèse, posons que la catholicité, en tant que système de valeurs et construit institutionnel, relève du structurel (au sens de Giddens, 1987) et est à la fois contraignante et habilitante dans le travail de construction de la référence et de la compétence identitaires basques, résultars d'une construction à la fois subjectivée et objecivée. Par référence, au sens de F. Dumont (Dumont, 1994), nous désignons la part de mémoire et d'utopie nécessaires à une affirmation identitaire mobilisable, sous une forme nationaliste ou pas. La référence renvoie à un niveau d'identification que Fernand Dumont voit comme un échelon supérieur à l'appartenance et à l'intégration. La religion intervient ensuite dans la construction de la compétence identitaire, dans les conditions d'émergence de ce savoir-faire spécifique lié à une conscience discursive et à une conscience pratique particulières; à la mobilisation de ressources et à la transformation des contraintes en ressources. La référence d'un côté, la compétence de l'autre : l'intervention du religieux dans la structuration de la motiva-

\footnotetext{
I Cet article reprend brièvement les principales articulations et conclusions d'une thèse de doctorat en science politique soutenue en novembre 2000 à l'IEP de Bordeaux (Itçaina, 2000). La thèse a bénéficié du soutien du Ministère di: la recherchc at d'l.usko lkaskuntza.
} 
tion identitaire prend deux formes indissociables mais nécessitant deux approches méthodologiques distinctes, qui constituent l'ossature de ce travail.

\section{Clergé et nation : catholicisme et construction de la référence identitaire}

Une première partie s'interroge sur la part du religieux dans la construction d'une référence collective associée à l'idée de nation basque. En considérant la nation et sa traduction politique, le nationalisme, comme autant de conflits d'exégèse, nous considérons que le religieux a contribué à diffuser et à installer dans les représentations sociales une interprétation particulière, une référence. Les mémoires des deux piliers institutionnels les plus visibles du religieux et de l'identitaire, clergé et mouvements nationalistes, sont confrontés ici. Une telle orientation, sous un aspect généraliste, implique au moins un double choix : nous ne traitons pas de la part de la religion locale (terme qu'avec W. A. Christian (Christian, 1997) nous préférons à celui, ambigu, de religion populaire) dans l'identité des Basques, sauf lorsque la routinisation de la religion locale sera perturhice par une relecture politique. En recentrant ensuite l'analyse sur les prêtres, nous excluons du même coup le personnel religieux féminin. objet, pourtant, d’un questionnement sociologique spécifique. La moindre visibilité en terrain basque de la portée politique des mobilisations religieuses féminines a déterminé ce choix. Concernant l'objet ainsi réduit. on observe la co-occurrence étroite de deux processus, sans qu'ill soit possible d'établir entre eux une relation de causalité : la construction d'un mouvement nationaliste/nationalitaire institutionnalisé d'une part, l'émergence du clergé basque comme acteur politique semi-autonome d'autre part. L'émergence du "clergé basque » comme vecteur d'action collective est antérieure à la première expression politique du nationalisme à la fin du XIX' siècle. Avec des traductions différentes des deux côtés de la frontière, la trajectoire historique du clergé a pu diffuser la référence d'un mode d'action alternatif à la construction des Etats-nations français et espagnol, sans qu'une telle attitude, d'abord motivée par des intérêts religieux, n'induise forcément un quelconque engagement nationaliste. Reste qu'en agissant contre l'Etat et en s'érigeant comme représentant légitime du peuple, le clergé basque a pu contribuer à diffuser une culture de la distance.

\section{Le fil rouge: le répertoire d'action collective du clergé basque.}

La périodisation d'une référence en construction s'est alignée sur les phases du nationalisme, en prenant comme fil rouge l'action collective du clergé. Il y a un répertoire spécifique de l'action cléricale basque, historiquement construit, et modulable en fonction des structures d'opportunité politiques. Cette façon d'agir ensemble, historiquement transversale, présente cinq caractéristiques (chapitre 1) 
Le mode de direction est marqué par des pratiques assembléistes et une dilution des leaders. Il y a un leadership informel au sein des groupes de prêtres mobilisés, mais sa visibilité est volontairement réduite au minimum dans le souci d’une image égalitariste.

b) Le mouvement est restreint aux prêtres de paroisse, aux religieux, aux aumôniers. La hiérarchie diocésaine apparaît comme occupant une position médiane entre autorités étatiques et clergé. Son statut de régulateur intègre pétit à petit une gestion institutionnalisée de la question identitaire. L'interaction entru liu hrerarchie et les groupes de prêtres ne peut être qualifiée de façon abrupte : en règle générale, cependant, la défiance mutuelle est de mise, sans pour autant que les écarts de position aboutissent nécessairement au conflit.

c) Les ressources de la mobilisation cléricale sont essentiellement de nature symbolique et réactivées par des groupes latents peu institutionnalisés. La création de groupes durables de prêtres et de chrétiens abertzale dans l'immédiat post-franquisme constitue à ce titre une innovation, mais ces structures ne sont réactivées que ponctuellement.

d) $\quad$ Il y a des degrés divers dans la protestation, qui va de la réunion de prière jusqu'à l'incendie volontaire de la prison, en passant par la pétition, la grève de la faim ou l'accueil aux clandestins. Ce répertoire est modulable : il s'est ajusté aux changements historiques en privilégiant telle ou telle forme d'action. Dans les années 1960 , le répertoire d'action est directement inspiré du renouveau mondial en la matière (grèves de la faim, occupations de locaux, grève des séminaristes, marches). La plupart des anciens savoir-faire, cependant, persistent : les actions concertées des prêtres prennent la forme de sermons, de pétitions, de réunions, et surtout d'un appel vers Rome, court-circuitant l'autorité diocésaine.

e) II y a un écart entre la réponse des destinataires formels de l'action (les autorités ecclésiastiques), généralement négative, et la réception forte de l'action dans les milieux politiques et sociaux.

Ces cinq caractéristiques se retrouvent de façon variable et combinatoire sur chacune des périodes, et contribuent à ériger un modèle spécifique de protestation. Par sa récurrence historique et sa dimension utopique, un tel registre a pu constituer une référence : au-delà des revirements idéologiques radicaux, une même manière de protester persiste. Ce répertoire constitue, pour une minorité du clergé, ce que S. Tarrow qualifie de cadre culturel de signifícation (cultural frame of meaning). Les prêtres protestant contre le régime franquiste en 1968 ont recours à un usage de la parole et de l'acte dont la genèse remontait aux guerres carlistes, mais ils le font au nom d'une idéologie diamétralement opposée à celle des curés légitimistes du XIX" siècle. Les savoir-faire pratiques se sont mieux transmis que les idéologies, instaurant ce que S. Tarrow nomme « une convention dans le contentieux (contention by convention): 
«L'action collective n'est pas sortie de la tête des organisateurs, mais elle est culturellement inscrite et communiquée. Les conventions apprises d'action collective font partie de la culture publique/politique (public culture) d'une société. Les groupes particuliers ont une histoire particulière - et une mémoire - de l'action collective. Les ouvriers savent comment faire grève, parce que des générations d'ouvriers ont fait grève avant eux; les Parisiens construisent des barricades parce que les barricades sont inscrites dans l'histoire des contentieux parisiens; les paysans s'emparent de la terre en utilisant les symboles que leurs aïeuls ont utilisés dans le passé » (Tarrow, 1994: 19). Les prêtres font des pétitions, des grèves de la faim, en appellent à Rome, parci: qu'ils conncissent cette façon de faire et parce que la structure des opportunités politiques les y pousse. Une telle récurrence n'implique en rien une position dominante de cette forme de protestation au sein de l'institution : la plus grande partie de l'Eglise fonctionne sur d'autres régimes de la parole et du symbolique, non évoqués ici, mais qui relèvent aussi d'une mémoire en construction. Le répertoire analysé est historiquement minoritaire, mạis périodiquement réactivé.

\section{Généalogie historique d'un répertoire.}

La récurrence historique d’un tel ripertoire a contribué à instaurer une mémoire de la distance vis-à-vis des constructions stato-nationales française et espagnole. La référence ainsi constituée se notirrit de la traduction partielle dans le champ politique (par les partis et organisations légitimistes, nationalistes et nationalitaires) des catégories religieuses de l'intransigeantisme, du libéralisme, de l'intégralisme et, dans une moindre mesure, du fondamentalisme. Ce modèle, historiquement dynamique, a connu quatre phases:

a

La réaction légitimiste : la défense du catholicisme intransigeant va de pair avec celle des droits historiques au Sud. Elle critique /'Thut mais contribue à une socialisation nationale française de la population au Nord. Dans tous les cas, la mobilisation carliste au Sud comme li: discours anti-républicain du clergé au Nord instituent selon des modalités différentes les conditions pour que ces territoires expérimentent une défiance vis-it vis d'une représentation fortement normative et prescriptive de l’Etat central (chapitre 2).

b) L'affirmation ethno-nationaliste : le nationalisme basque évolue d'un intégrisme racial vers une démocratie-chrétienne ethnique, qui bénéficie du soutien des prêtres mais est critiqué par la hiérarchie de l'Eglise. Il se diffuse de façon marginale au Nord, où sa réduction à une forme culturaliste est due en grande partie à l'action du clergé (chapitre 3).

L'émergence nationalitaire : l'affirmation ethno-territoriale se sécularise, au Nord comme au Sud. Cependant, le clergé, loin de disparaître de la scène politique, ajuste 
son répertoire d'action à la nouvelle donne. Le recours à une théologie de la libération réajustée au contexte basque est une issue théorique opérationnelle aux quescionnements liés à la sécularisation (chapitre 4).

L'instauration d'une démocratie libérale en Espagne et l'institutionnalisation progressive des mouvements identitaires côté français marginalisent le répertoire d'action du clergé basque. En revanche, d'autres secteurs ecclésiaux (en premier lieu l'épiscopat) réinvestissent l'espace public en prononçant en particulier des expertises éthiques sur la paix. Côté français, l'enjeu identitaire est traité par l'institution, mais de façon dissimulée et en respectant mieux la séparation des domaines politiques et religieux (chapitre 5).

\section{Socialisation religieuse et construction de la compétence identitaire}

\section{De la référence à la compétence:}

vers une approche complémentaire.

Deux questions restent en suspens: les mobilisations identitaires se résurnent-elles à l'action collective nationaliste? L'influence du religieux se ressent-t-elle uniquement au travers des actions collectives du clergé? Afin d'y répondre, la deuxième partie de la thèse a été construite autour de lis nution de compétence. La première partie a analysé la construction de la référence mobilisatrice sur le temps long en se centrant sur les organisations et les acteurs les plus visibles des deux pôles : le clergé et les nationalistes. Or l'interaction entre le religieux et l'identitaire est loin de se résumer à cette visibilité. L'analyse historique doit être complétée par une sociologie de la construction d'une identité militante : celle-ci est d'abord une prise de rôle institutionnelle qui implique le réinvestissement d'un savoir-faire appris puis réajusté, sans qu'une telle socialisation de base n'induise un quelconque déterminisme. Cette partie est chronologiquement plus restreinte (deuxième moitié du XXe siècle), mais thématiquement plus large, dans la mesure où l'expression de la militance sera recherchée dans des domaines ne relevant pas stricto sensu d'une action nationaliste objectivée (c'est-à-dire orientée vers une coïncidence exacte entre l'Etat et la nation) mais contribuant tout autant à construire une référence identitaire dans des secteurs plus spécialisés, abusivement situés hors du politique.

Une socialisation religieuse forte a pu contribuer à générer des prédispositions pour l'apparition d'un militant généraliste. Celui-ci s'affranchira progressivement de la tutelle religieuse et se mutera en expert avec la spécialisation des tâches inhérentes à l'institutionnalisation de la mobilisation identitaire. La construction de la compétence a été analysée dans un moment historique particulier, celui d'un retrait institutionnel et social rapide et inédit de 
I'Eglise catholique en Pays basque, retrait doublé d'une recomposition importante de Ia mobilisation identitaire qui, loin de se limiter à ses seules expressions nationalistes/nationalitaires, a aussi emprunté d'autres voies. A partir d'une approche critique à la fois des théories classiques de la sécularisation du politique et des percuptions totalinantes de la culture (chapitre 6), nous avons pu repérer la permanence des effets de la socialisation catholique :ur trois sites d'observation

a)

La culture: l'objectivation d'une culture basquic autonome, centrée autour de l'enjeu linguistique, s'est effectuée en grande partie dans les institutions religieuses, en particulier dans le contexte ascétique et utopique des communautés monastiques ${ }^{2}$ (char pi(re 7$)$.

b) L'économie: les formes identitaires de l'économie (syndicalisme agricole et coupérativisme) trouvent une source de leur genèse dans l'Action catholique et les institutions d'enseignement catholiques ${ }^{3}$ (chapitre 8 ).

c) La socialisation militante: l'expérience institutionnelle d'une socialisation religieuse forte a pu contribuer à construire un ethos militant particulier, suivant trois modalités biographiquement repérables ${ }^{4}$ (à partir d'une trentaine de récits de vie d'anciens prêtres et d'anciens séminaristes) : continuité, séparation, entre-deux relativiste (chapitre 9).

L'institution catholique a ainsi contribué. par la socialisation aux valeurs et aux compétences, à former un militant idencitaire porteur d'unc éthique particulière, à la fois généraliste et spécialiscic. mais toujours orientée vers l'engagement. Le recul institutionnel de I'Tglise catholiqu' n'élimine pas les effets culturels d'une socialisation religieuse, et bon nombre d'objectivutions et d'institutionalisations qualifiées de culturelles, économiques ou politiques "basques" pelvent être relues comme des traductions de valeurs, de croyances et de savoir-faire partiellement acquis au sein de l'institution catholique. Une socialisation au sein de l'institution religicuse est le lieu et le temps où s'effectuent l'apprentissage et la construction des définitions rivales de la réalité. Eille agit en ce sens à la fois comme ressource et contrainte dans l'objectivation préalable aux rôles institutionncls futurs. La socialisation catholique a de l'importance dans l'émergence d'une première fịgure du militant, puis dans sa transformation en expert en matière de corps raréfié de connaissances, au terme d'un proces-

2 On s’inspire ici des analyses de Jean Séguy sur la dimension utopique du monachisme (Séguy. 1980), de l'approche de l'institutionnalisation de la culture chez M. de Certeau (De Certeau. 1980) et de l'anthropologie de l'écriture de J. Goody (Goody, 1977).

3 L'approche weberienne classique (Weber, 1964) est ici combinée à l'approche de l'économie sociale et de la sociologie religieuse chez $\mathrm{H}$. Desroche (Desroche, 1968) et à des études de cas contemporaines des ressources sociales et culturelles de telle forme spécifique de développement local (Palard, 1999).

4 Sur les problèmes posés par la méthodologic des récits de vie dans l'analyse de la socialisation. voir (Dubar, 1997). 
sus de spécialisation et de division du travail (on distingue désormais le politique, le culturel. l'économique) qui affecte l'ensemble des mobilisations identitaires basques.

Une compétence partagée enire croyances, valeurs et savoir-faire.

La compétence identitaire basque en construction s'articule sur des croyances, des valeurs et des savoir-faire spécifiques, partiellement reliés à une socialisation religieuse des acteurs. Il y a un système dynamique de valeurs identitaires, au sens de principes spécifiques de régulation de l'action. Nous parlons bien de valeurs et non pas d'idéologies. En réservant le terme d'idéologie aux formulations spécifiquement nationalistes, nous soulignons bien que les valeurs qui guident partiellement les actions des militants/experts identitaires ne reposent pas forcément sur des théories formalisées. Si l'on consent, avec R. Boudon, à définir les idéologies comme « des doctrines reposant sur des théories scientifiques, mais sur des théories fausses ou douteuses ou sur des théories indûment interprétées, auxquelles on accorde une crédibilité qu'elles ne méritent pas » (Boudon, 1986 : 45), il nous faut alors réserver l'emploi de ce terme, comme nous l'avons fait dans la première partie, aux expressions postulant l'existence d'une nation basque aspirant à une souveraineté politique. En se centrant sur des domaines projetés par le sens commun hors du politique, cette deuxième partic a quitté le clomaine de l'idéologie pour s'inscrire dans celui, plus large et moins repérable, des valeurs.

Or, c'est précisément sur ce terrain que la relation au religieux est la plus féconde. Si, par exemple, au lieu de ne voir dans l'expérience coopérative basque qu'une simple transposition économique de l'idéologie nationalitaire, on la considère plutôt comme une tentative d'application de préceptes comme la solidarité, la participation et le développement endogène, alors la comparaison avec les référents religieux devient opératoire (chapitre 8). De même si, par l'autonomisation de la culture, nous repérons autre chose que l'institutionnalisation d'un nationalisme culturel ou ethnique, mais bien plus profondément la fixation sous une forme graphique d'une culture dispersée (aux sens de J. Goody (Goody, 1977) et de F. Dumont (Dumont, 1995), on voit mieux sur quels processus longs largement mis en place par l'institution catholique se greffe l'objectivation culturelle (chapitre 7). Enfin, lorsque les acteurs, au terme d'une révision de leur itinéraire, situent les ponts entre socialisation catholique et engagement identitaire au niveau de l'apprentissage de l'engagement, de l'altruisme voire de la méfiance vis-à-vis des institutions, le discours porte sur les valeurs et non pas sur l'idéologie (chapitre 9). D'où, en particulier, notre réserve au moment de traduire le terme d'abertzale par nationaliste/nationalitaire. D'où, plus généralement, le pluriel accolé à «identité basque » dans ce travail. Lorsque les acteurs se disent abertzale, ils entendent bien désigner l'ajout d'une conscience politique à une conscience culturelle, mais sans pour autant associer automatiquement une telle aspiration à telle ou telle théorie formalisée et explicitement reconnue par les partis et organisations. Se dire abertzale est plutôt une façon d'affirmer que tout est lié : 
l'économique, le culturel ne peuvent fonctionner sans le politique, et vice versa. Une vision globale, qui montre la persistance du premier modèle du militant généraliste, et qui s'ajuste à l’exigence de réalisation connotée par la référence aux valeurs

L'approche par les valeurs comporte un risque, que nous avons souligné à plusieurs reprises, en particulier grâce aux analyses d'A. Giddens et de J. Lagroye (Lagroye, 1997a, 1997b) : celui de se limiter à la conscience discursive des acteurs, d'arrêter l'analyse à ce que les acteurs disent d'eux-mêmes et de leur action, et de confondre ce qui est dit et ce qui est fait ${ }^{5}$. Même si l'on reste dans la sphère des valeurs, une telte approche ne retiendra que les valeurs positives verbalisées par les militants. Or, derrière l'engagement, la solidarité, la défense des faibles (des minorités sociales ou culturelles), on trouve la permanence d'autres principes non verbalisés, ou implicitement dissimulés : le refus de transiger sur certains points. un recours fréquent au conflit comme mode de fonctionnestent, enfin une sur-détermination de l'action précisément par les valeurs verbalisées. Encore une fois, les variations dans le degré d'engagement - y compris dans sa dimension physique et violinte - induisent un rapport distinct à ces valeurs implicites. En quittant cette fois le monde des principes, nous avons vu que l'apprentissage en milieu religieux pouvait conduire à l'intériorisation de savoir-faire pratiques réinvestis ensuite dans l'action identitaire (pratiques assembléistes et participatives. initiatives par la bas, etc.). En termes urganisationnels, l'exemple de la fabrication culturelle à l'œuvre dans les revucs ir.sues des institutions religieuses et celui des structures syndicales et coopératives reliées à l urigine à des mouvements d'Action catholique et à des prêtres-animateurs illustre ce travail ù traduction de savoir-faire intériorisês. Les itinéraires individuels témoignent aussi d’une pré-disposition au travail militant acquise en partie au sein de l'institution. Ces processus ne sont pas toujours verbalisés, ils relèvent de la conscience pratique des acteurs, mais sont des composants essentiels de leur compétence, schématiquement repris ici :

\footnotetext{
5 Même si, objecteraient les tenants de la philosophie analytique, «dire, c'est faire », surtout lorsque les conditions d"un énoncé performatif sont réunies, ce qui est particulièrement crucial en matière religieuse (Austin, 1970).
} 


\begin{tabular}{|c|c|c|c|}
\hline \multicolumn{4}{|c|}{ Composantes de la compétence } \\
\hline & Croyances & Valeurs & Savoir-faire pratiques \\
\hline $\begin{array}{l}\text { Socialisation } \\
\text { catholique }\end{array}$ & $\begin{array}{l}\text { Croyances } \\
\text { normatives } \\
\text { Il y a des vérités ulti- } \\
\text { mes (dogmes) } \\
\text { L'institution } \\
\text { catholique a une } \\
\text { légitimité spécifique, } \\
\text { que n'a aucune } \\
\text { autre institution. } \\
\text { Croyances positives } \\
\text { Efficacité } \\
\text { symbolique des rites } \\
\text { L'institution dispense } \\
\text { des rétributions } \\
\text { matérielles et } \\
\text { symboliques }\end{array}$ & $\begin{array}{l}\text { Valeurs implicites: } \\
\text { Refus du relativisme } \\
\text { absolu } \\
\text { Refus de l'individua- } \\
\text { lisme }\end{array}$ & $\begin{array}{l}\text { Apprentissage de } \\
\text { l'autonomie relative } \\
\text { au sein d'une institu- } \\
\text { tion très codifiée et } \\
\text { très prescriptive } \\
\text { Apprentissage de la } \\
\text { transgression des } \\
\text { rôles comme mode } \\
\text { d'affirmation } \\
\text { identitaire } \\
\text { Manipulation de } \\
\text { I'abstraction et de la } \\
\text { contradiction } \\
\text { comme mode de } \\
\text { fonctionnement } \\
\text { Formation d'un mili- } \\
\text { tant généraliste : une } \\
\text { vision globale déter- } \\
\text { mine l'action secto- } \\
\text { rielle }\end{array}$ \\
\hline $\begin{array}{l}\text { Socialisation iden- } \\
\text { titaire basque }\end{array}$ & $\begin{array}{l}\text { Croyances norma- } \\
\text { tives } \\
\text { Le Pays basque } \\
\text { existe en tant que } \\
\text { peuple/pays (herr) } \\
\text { Croyances } \\
\text { positives } \\
\text { Une action collec- } \\
\text { tive est nécessaire } \\
\text { pour sa survie }\end{array}$ & $\begin{array}{l}\text { Valeurs verbalisées: } \\
\text { Solidarité, altruisme } \\
\text { Engagement } \\
\text { Valeurs implicites: } \\
\text { Refus du } \\
\text { relativisme absolu } \\
\text { Refus de } \\
\text { l'individualisme } \\
\text { Refus de transiger } \\
\text { sur certains points }\end{array}$ & $\begin{array}{l}\text { Formes d'organisa- } \\
\text { tions participatives } \\
\text { Pratiques } \\
\text { assembléistes } \\
\text { Méfiance vis-à-vis } \\
\text { des institutions } \\
\text { trop normatives } \\
\text { Familiarité avec le } \\
\text { débat d'idées } \\
\text { Spécialisation et } \\
\text { division du travail } \\
\text { identitaire : le mili- } \\
\text { tant devient expert, } \\
\text { le sectoriel s'auto- } \\
\text { nomise. }\end{array}$ \\
\hline
\end{tabular}


Les limites du recours au religieux.

En montrant que des «ponts » existaient entre les croyances, les valeurs et les savoirfaire catholique et identitaire-basque, nous n'avons pas voulu signifier que la compétence du militant/expert était entièrement déterminée par le religieux. La variété des regards que portent les acteurs sur leur propre itinéraire interdit d'instaurer une relation automatique. Ce qui, en revanche, ne laisse que peu de place au doute, c'est qu'une socialisation religieuse poussée comme a pu l'être un long séjour au séminaire ou, dans une moindre mesure, au sein de l'Action Catholique, est un choc biographique qui, en retour, structure les changements d'identités ou alternations, au sens de P. Berger et T. Luckmann (Berger et Luckmann, 1986). Bien sûr, les circonstances historiques considérées ici sont propices à ce que l'évolution identitaire prenne un tournant dramatique : un régime national-catholique vit ses dernières années en Espagne, marquées par un raidissement répressif inédit depuis la fin de la guerre civile. Au Nord, la structuration du mouvement nationalitaire et l'arrivée des réfugiés du Sud imposent un sens politique inédit aux expressions identitaires. L'Eglise, de son côté, est dans une situation ambivalente : elle c...t en pleine remise en question interne et externe mais elle occupe encore en Pays basque une position sociale prédominante. Nous rejoignons ici l'interp ctation de la culture comme «boîte à outils» (tool kit) d'Ann Swidler (Swidler, 1986) pour qui la visibilité de l'effet de la culture sur les compétences (skills) engagées dans l'action et sa reproduction est beaucoup plus importante lors de périodes d'instabilité.

Le militant en construction, somme, so trouwe dans une situation assez facile à interpréter. La plupart des témoignages sollicités ici le soulignent : à l'époque (avant la relative sécularisation interne des institutions catholiques), la situation est perçue comme simple. Il y a lé bloc des institutions dominantes d'un côté, où l'on range l'Eglise, le régime politique et les notables, et un peuple/pays qui souffre de l'autre. D'autre part, l'expérience d'une socialisation religieuse forte est une expérience politique : l'institution est vécue de l'intérieur, avec ses luttes de pouvoir, ses conflits de valeurs et d’idéologies. Le décalage entre le mode de fonctionncrinent d'une institution catholique et l'évolution sociale donne une portée conflictuelle à là moindre contestation. Il pousse surtout les membres de l'institution à objectiver leur situation et à l'interpréter de manière politique. c'est-à-dire en termes de rapports de pouvoir.

Autrement dit, dans ce contexte, l'acteur peut assez rapidement s'estimer et être estimé compétent en matière politique, contrairement, par exemple, à la génération de ses parents. Une analyse inspirée de P. Bourdieu (Bourdieu, 1979) verrait là une réhabilitation de la compétence politiqute provoquée par un changement des positions des agents sociaux dans le champ des rapports de classes. A cette réserve riè yu une socialisation cathnlique forte ne peut pas être interprétée comme un changement de position dans le champ des rapports de classe : si les futurs militants ruilixent l'existence de classes sociales lorsqu'ils sont à l'inté- 
rieur de l'institution catholique, cela ne signifie pas qu'ils accèdent forcément eux-mêmes à une position sociale plus élevée que celle de leur milieu d'origine. La construction de la compétence passe davantage par l'expérience de rôles successifs très différents pourtant associés à des positions institutionnelles très proches que par un changement attribué de statut social. La dramatisation historique particulière associée à l'expérience vécue de l'institution contribue à construire la compétence identitaire de chacun, qui se traduit ensuite en termes organisationnels et institutionnels.

Les deux réponses (celles de la référence et de la compétence) se veulent complémentaires : si la première fournit à « la religion pour mémoire " (Hervieu-Léger, 1993) la profondeur historique qu'elle sous-entend, la deuxième entend replacer l'acteur au cour de ces processus de recomposition. La part du religieux dans la socialisation militante est effective mais non déterministe. L'acteur est libre de ses choix, mais il doit renégocier en permanence ses affitiations identitaires précédentes. II ne peut, en l'occurrence, faire totalement abstraction de certaines prédispositions qui, elles, n'étaient que partiellement choisies au cours des différentes étapes biographiquè. Autrement dit. l'exemple du religieux en terrain basque confirme une fois de plus, à un niveau plus général, la vacuité de l'opposition entre approches objectivistes et approches phénoménologiques et individualistes qui dénonce P. Bourdieu (Chartier, Bourdieu, 1989 : 54). L'action militante identitaire est tout autant motivée par l'accès à des objectifs concrets (le motif en-vue-de) que par une réponse à une série de prédispositions (le motif parce-que) (Schütz, 1998 : 56-59) qui funt que l'objectif n'est pas forcément celui affiché sous une forme rationnelle. Or une socialisation religieuse a pu influer sur ces deux dimensions, dont l'exploration plus approfondie relèverait davantage de la psychosociologie ou d'une sociologie phénoménologique poussée que de la sociologie politique esquissée ici.

Au total, de façon théorique et empirique, cette recherche propose un cheminement allant d'une sociologie historique des institutions vers les constructions identitaires individuelles. En montrant comment le religieux a contribué à former la compétence des experts identitaires tout en l'inscrivant à l'horizon d'une référence collective, ce "cinquième récit » s'assigne surtout une mission exploratoire pour des recherches futures. C'est en ce sens qu'il prétend au statut d'innovation relative vis-à-vis d'une thématique déjà partiellement exploitée. 


\section{Bibliographie indicative}

\section{Méthodologie, religion et construction sociale de l'identité}

John Langshaw AUSTIN, Quand dire, c'est faire. Paris, Seuil, 1970 [How to do things with words, Oxford University Press, 1962].

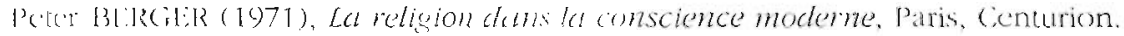

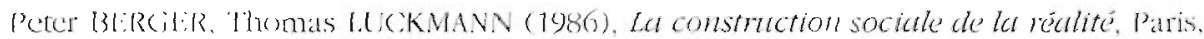
Méricliens Klincksieck ITbe social constraction of reality. A treatise in the sociology of knouleclese, (iarden (ity. N.Y., I) (xul)lediay. 1966).

Raymond BOUDON (1986), L'idéologie ou l'origine des idées reçues, Paris, Fayard, 1986 Danièle HERVIEU-LEGER (1993), La religion pour ménoire, Paris, Cerf.

Pierre BOURDIEU (1979), La distinction, critique sociale du jusement, Paris, Minuit.

Pierre BOURDIEU, Roger CHARTIER (1989), « Gens à histoires, gens sans histoires. Dialogue entre Pierre Bourdieu et Roger Chartier », Politix, 6, p. 53-60.

MicheI DE CERTEAU (1980), La culture au pluriel, Paris, Christian Bourgeois.

Henri DESROCHE (1968), Sociolusies religieuses, Paris, PUF.

Claude DUBAR (1991), La socialisation. Construction des identités sociales et professionnelles, Paris, Armand Colin.

Fernand DUMONT (1994), Le lieu de l'homme, la culture comme distance et mémoire, Québcc. Bibliothèque québecoise [Montréal, Hurtubise, 1968].

Fernand DUMONT (1995), Le son de la culture, Montréat, Typo.

Anthony GIDDENS (1987), La constitution de la société, éléments de la théorie de la structuration. Paris, PUF [The constitution of societ: : outline of the theory of structuration. Cambridge, Polity press, 1984].

Jack GOODY (1977), La raison graphique, la domestication de la pensée sauvage. Paris, Minuit.

Jacques L.AGROYE (1997a), Sociologie politique, Paris, Presses de Sciences Po, Dalloz.

Jacques LAGROYE (1997b), «On ne subit pas son rôle. Entretien avec J. Lagroye », Politix, 38,1997, p. $7-17$.

Jacques PALARD (1999), «Structures sociales, traditions culturelles et innovation industrielle dans la Beauce québécoise ». Sciences de la société, 48, p. 137-148.

Jean SEGUY (1980), « La socialisation utopique aux valeurs ", Archives de sciences sociales des religions, $50(1)$, p. 7-21.

Ann SWIDLER (1986), «Culture in action: symbols and strategies », American sociological review, 51 (2), p. 273-286.

Alfred SCHÜTZ (1998). Eléments de sociologie phénoménologique, Paris, L'Harmattan, p. $56-59$.

Sydney TARROW (1994), Power in movement, social movements, collective action and politics, Cambridge. Cambridge University Press. 
Max WEBER (1964 [1904]), L'érhique protestante et l'esprit du capitalisme, Paris, Plon.

\section{Religion et construction identitaire en Pays Basque :}

Joxe AZURMENDI (1984), El hombre cooperativo, pensamiento de Arizmendiarrieta, Lankide Aurrezkia, Editorial Franciscana Arantzazu (Jakin).

Pierre CHARRITTON, Le droit des peuples à leur identité. L'évolution d'une question dans I'histoire du christianisme, Montréal, Fides, 1979

William A. CHRISTIAN (1997), Las visiones de Ezkioga. La secunda República y el reino de Cristo, Barcelona, Ariel.

Philippe FABA:; (1999), Asper's de la vie relivicusc dans le diocèsc de Bayonne (1905-1965). thèse en Histoire contemporaine, Bordeaux III

William FOOTE WHYTE, Kathleen KING WHYTE (1989), Mondragón : más que una utopía, Donostia, Txertoa [Making Mondragón, Cornell University Press, 1988].

Xabier ITCAINA (2000), Catholicisme et identités basques en France et en Espagne. La construction religieuse de la référence et de la compétence identitaires, Thèse pour le doctorat en science politique, IEP Bordeaux (dir. Jacques Palard).

Xabier ITCAINA, « Catholicisme, identité collective et affinités électives. Les coopératives basques comme groupements volontaires utopiques ». Social Compass, revue internationale de sociologie religieuse, Louvain, (à paraître) 2002/3 ou 2003/1.

Paulo IZTUETA. (1981), Sociología del fenómeno contesiatorio del clero vasco: 1940-1975 (análisis de las causas de la radicalización del clero vasco en el periodo 19401974), Bayonne, Donostia, Elkar.

Paulo IZTUETA (1987), «Eliz mugimenduak Euskal Herrian : Baserri gaztediaren eta Euskaldun Gazteriaren kasua », in Rafael LOPEZ-ATXURRA (dir.), Euskal Herriaren historiaz, III. Kultura eta portaera erlijiosoak Euskal Herrian zehar, Bilbao, EHU-UPV, p. 183-203

Sharryn KASMIR (1996), The myth of Mondragón. Cooperative, politics and working-class life in a Basque town, Albany, NY, State university of New York Press.

Denis LABORDE (dir.) (1998), La question basque, Paris, L'Harmattan.

Xabier LASA GORRAIZ (1998), Manex Erdozaintzi-Etxart, biziza eta lan teologikoen azterketa, 2 t., lizentziatura tesina, teologia fakultatea, Gasteiz.

Pierre LARZABAL (1978), Nere mendixkatik. ETA'ko gudariez apez bat mintzo, Donibane Lohitzune, Dizkola. 
Philippe MAYTE (1992), L'Eskualdun Gazteria (JAC, JACF, MRJC) des années 1930 à la fin des années 1970, T.E.R., maîtrise d'histuire contemporaine, Bordeaux III.

Michel ORONOS (dir.) (1982). Jalons pour une histoire de l'Eglise catholique en Pavs Basque nord de 1965 à 1977, Bayonne.

Michel ORONOS (1989), "Contribution à l'histoire de l'Eglise catholique en Pays Basque ». Hommage au Musée Basque, Bayonne, Société des Amis du Musée Basque, p. 297 309 .

Jean-Baptiste ORPUSTAN (1980), « Rôle et pouvoirs de l'Eglise », dans Pierre BIDART (dir.), La nouvelle société basque, Paris, L'Harmattan, p. 108-158

Alfonso PEREZ-AGOTE (1986), "The role of religion in a symbolic conflict : religion and the Basque problem ». Social compass, revue internationale de sociologie religieuse, 33 (4), 1986, p: 419-435.

AIfonso PEREZ-AGOTE (1990), Los lugares sociales de la religión. La secularización cie la vida en el País Vasio. Madrid. CIS.

Felix PLACER UGARTE (1998), Creer en Luxal Herria, la expriencia creyente de las Comunidades cristianas populares y de la Coordinadora de Sacerdotes de Eliskal Herria, 1976-1996, Bilbao, Herria 2000 Eliza.

José Antonio PAGOLA (comp.) (1995), Una ética para la paz, los obispos del País Vasco. 1968-1992, Donostia, Idatz.

José María SETIEN (1998), Obras complezas. I. Dios, política, pas. Donostia, Idatz.

Martine SISTIAGUE (1999), ELB, Ipar Euskal Herriko laborarien oihartzuna. Bayonni, Gatuzain.

Julen URKIZA (1995), Elizaren historia liuskal Herrian. l. Ikerlan materialak, Markina, El Carmen.

Joseba ZULAIKA (1988), Basque violence, metaphor and sacrament. Reno, Las Vegas. University of Nevada Press.

Joseba ZULAIKA (1996), Del Cromañon al Carnaval, los Vascos como museo antropológico. Donostia, Erein. 